\title{
Trimodality Bladder Preservation Therapy for Muscle- Invasive Bladder Cancer: Mansoura Experience
}

\author{
Eman Awad Abd Allah, Fatma Mohamed Farouk Akl , Seham Elsayed-Abd-Alkhalek \\ Clinical Oncology \& Nuclear Medicine Department, Mansoura University, Mansoura, Egypt \\ Email address: \\ mlak2003@yahoo.com(E. A. A. Allah), fatmaak1@yahoo.com(F.M. F. Ak1), seham732000@yahoo.com(S. Elsayed-Abd-Alkhalek) \\ ${ }^{*}$ Corresponding author
}

To cite this article:

Eman Awad Abd Allah, Fatma Mohamed Farouk Akl, Seham Elsayed-Abd-Alkhalek. Trimodality Bladder Preservation Therapy for MuscleInvasive Bladder Cancer: Mansoura Experience. Cancer Research Journal. Vol. 7, No. 1, 2019, pp. 1-7. doi: 10.11648/j.crj.20190701.11

Received: December 7, 2018; Accepted: December 19, 2018; Published: January 24, 2019

\begin{abstract}
Background \& objective: Bladder preservation therapy (BPT) using a trimodality approach represents an alternative option to cystectomy inmuscle-invasive bladder cancer (MIBC) patients, also a treatment option in non-cystectomy candidates. The objective of this study was to evaluate BPT using a trimodality approach composed of maximum TURBT, neoadjuvant chemotherapy, followed by chemoradiotherapy, regarding the overall survival (OS), progression free survival (PFS), locoregional progression free survival (LPFS) and treatment toxicity. Patients \& methods: This prospective study involved 47 patients with pathologically proven MIBC (T2-T4a N0M0). The study involved muscle invasive bladder cancer patients who refused or were not cystectomycandidates. Patients enrolled received neoadjuvant 3cycles of Gemcitabine/Cisplatin, each cycle was every 21 days. Gemcitabine at $1000 \mathrm{mg} / \mathrm{m}^{2}$ on days $1 \& 8$ and cisplatin at $70 \mathrm{mg} / \mathrm{m}^{2}$ on day1, followed by Concurrent chemordiotherapy with cisplatin weekly $\left(40 \mathrm{mg} / \mathrm{m}^{2}\right)$. Radiation therapy included the whole bladder by 3D conformal planning to a dose of 64Gy/32Fxs. Results: Of the 47 patients, $25(53.2 \%)$ patients expressed complete response (CR), while 22(46.8\%) patients had incomplete response. The 4-year OS, PFS, and LPFS rates were $48 \%$, $38 \%$, and $42 \%$, respectively. Acute genitourinary (GU) toxicity of Grade 1 and 2 occurs in $54 \%$ and $24 \%$ of patients, respectively, while acute gastrointestinal (GI) toxicity (colic \&diarrhea) of Grade 1 and 2 occurs in $27.7 \%$ and $10.6 \%$ of patients, respectively. Conclusion: For MIBC patients who are non-cystectomy candidates, or who are motivated to maintain their bladders, trimodality bladder preservation therapy (BPT) can be considered as an effective alternative to radical cystectomy.
\end{abstract}

Keywords: Bladder Cancer, Neoadjuvant Chemotherapy, Concurrent Chemoradiotherapy, Trimodality Treatment, Bladder Preservation

\section{Introduction}

Bladder cancer is the commonest malignancy of the urinary system, with 79,000 new cases and 17,000 deaths in the United States annually. Worldwide, bladder cancer accounts for approximately 540,000 new cases and 188,000 deaths [1]. In Egypt, it constitutes $6.94 \%$ in both sexes in the period between 2008-2011 [2].

Nearly 75 percent of new bladder cancer diagnoses are earlystage and have not yet invaded the muscular layer of the bladder wall with the remaining 25 percent have muscle-invasive bladder cancer (MIBC) [3].

The gold standard for MIBC was radical cystectomy (RC) with pelvic node dissection [4]. Radical cystectomy has an impact on patients' quality of life (QOL) with genitourinary or sexual dysfunction [5]. Outcomes of bladder function and QOL in patients who received bladder preserving treatment showed $75 \%$ of patients maintaining their native bladder function and $59 \%$ with satisfactory sexual life [4].

Modern oncologic therapies are increasingly driven towards organ preservation and maximizing functional outcomes while maintaining treatment efficacy. Although not the standard of care, BPT continued to evolve for patients refusing or considered as non cystectomy candidates [6].

BPT with trimodal approach is an alternative to RC for MIBC selected patients, and who are not considered as cystectomy candidates. It includes a maximum transurethral resection of the 
bladder tumor (TURBT), followed by concurrent chemoradiotherapy, which offers patients a chance to maintain their bladders, and reduce the potential morbidity \& mortality associated with RC [7].

The English National Comprehensive Cancer Network Guidelines include BPT with chemoradiotherapy after maximum TURBT as an alternative treatment to RCin MIBC [8]. The European Association of Urology Guidelines states that multimodal PBT can be an alternative option in selected, non cystectomy candidates [9].

No large randomized trials compared bladder preserving treatment \& radical cystectomy in MIBC. However, available trials recorded comparable results between BPT and RC [10, $11]$.

A systematic review and meta-analysis of available trials assessing bladder preserving therapy usingTURBT followed by chemoradiotherapy in MIBC patients, detected complete response in $78 \%$ of patients, with 5-year OS of $56 \%$ [12].

A case controlled study showed comparable results between RC and chemoradiotherapy in MIBC. Thirty three patients treated with chemoradiotherapy due to poor performance status for surgery were matched with patients treated with RC of similar age. The 5 year DFS \& OS for $\mathrm{RC}$ and chemoradiotherapy were $63.2 \%$ vs $54 \%$ and $54.8 \%$ vs $56.6 \%$, respectively [13].

Long term results of trimodality therapy (TMT) are very good and comparable to $\mathrm{RC}$, with improving complete response rates with time (86\% in 2010-2013). This has been shown by both the Princess Margaret hospital experience and the Massachusetts general hospital experience. There is similar disease specific survival (DSS) in both TMT and RC ranging from $66 \%$ in 5 years to $59 \%$ in 10 years. Overall survival (OS) is also similar with $57 \% 5$ year and $39 \% 10$ year $[14,15]$.

The objective of this study was to evaluate BPT using a trimodality approach composed of maximum TURBT, neoadjuvant chemotherapy, followed by chemoradiotherapy, regarding the overall survival (OS), progression free survival( PFS), locoregional progression free survival (LPFS) and treatment toxicity.

\section{Patients\& Methods}

\subsection{Study Design}

After acceptance of the Mansoura Faculty of Medicine, institutional research board MFM IRB, this prospective trial was performed at the Clinical Oncology \&Nuclear Medicine Department, Mansoura University, from Feb 2013 to June 2017, 47patients with pathologically proven muscle invasive urothelial bladder carcinoma (T2-T4a N0M0) were enrolled. The study involved patients who refused radical cystectomy or were medically unfit for surgery or presented with unresectable disease.

\subsection{Patients Selection and Eligibility}

Eligibility criteria were; Eastern Cooperative Oncology Group Performance Status (ECOG PS) 0-2, T2-T4a Bladder
TCC, no hydronephrosis, those had solitary tumor with limited CIS. White blood cell count $\geq 3500 / \mu \mathrm{L}$; platelet count $\geq 100,000 / \mu \mathrm{L}$; hemoglobin $\geq 10 \mathrm{~g} / \mathrm{dl}$; hepatic function (AST, ALT $<2$ xupper normal limit (UNL), total bilirubin $\leq 1.5 \mathrm{X}$ UNL; and renal function (serum creatinine $\leq 1.5 \mathrm{mg} / \mathrm{dl}$, and creatinine clearance of at least $60 \mathrm{ml} / \mathrm{min}$ ). Patients with major comorbidities, like active infection, significant arrhythmia, or heart failure, were not eligible. All expected benefits and side effects of treatment were explained to all patients and they signed informed consents before enrollment.

All patients underwent cystoscopic examination, biopsy from bladder mass, CT and or MRI abdomen \&pelvis and CT chest. Bone scan was done only if there were symptoms or elevation of alkaline phosphatase. All patients underwent maximal TURBT.

Patients enrolled received neoadjuvant 3 cycles of Gemcitabine/Cisplatin, each cycle was every 21 days. Gemcitabine at $1000 \mathrm{mg} / \mathrm{m}^{2}$ on days $1 \& 8$ and cisplatin at $70 \mathrm{mg} / \mathrm{m}^{2}$ on day1, followed by Concurrent chemordiotherapy with cisplatin weekly $\left(40 \mathrm{mg} / \mathrm{m}^{2}\right)$. CT simulation in the supine position, using $5 \mathrm{~mm}$ slice thickness was performed prior to planning, with empty bladder. The CTV included the GTV (primary tumor and extravesical spread) and the whole bladder, PTV included the CTV plus1.5-2 $\mathrm{cm}$ around the bladder. Delineation of target volume and organs at risk was done. All patients were planned by 3D-Conformal radiotherapy(3D-CRT) with ELEKTA Linear Accelerator, and precise treatment planning software. The photon energy used was $6 \mathrm{MV}$ and15 MV. Dose was $64 \mathrm{~Gy} / 32 \mathrm{Fxs}$, with standard fractionation (2 Gy/Fraction /day). Chemotherapy Toxicity was assessed and graded according to the Common Terminology Criteria for Adverse Events (CTCAE) version 4.

\subsection{Treatment Evaluation \& Follow Up}

During treatment, weekly $\mathrm{CBC}$, serum creatinine and clinical assessment for any side effects related to radiation or chemotherapy were performed, radiation reactions were reported and graded according to the Radiation Therapy Oncology Group (RTOG) toxicity criteria. After completion of chemoradiotherapy by 2 months, abdominopelvic MRI and cystoscopic examination were done for evaluation of treatment response. Patients were followed up by abdominopelvic MRI, cystoscopic examination and urine cytology every 3-6 months for 2 years, every 6 months for the subsequent 3 years and then annually. Bone scan and other radiologic investigations were done when clinically indicated. Salvage cystectomy was the treatment option for residualorrecurrent disease.

\subsection{Statistical Analysis}

Data were entered and analyzed using SPSS software (version 21). Qualitative data were expressed as count and percent. Quantitative data were tested for normality using KolmogorovSmirnov and Shapiro-Wilk's test with data being normally distributed if $p>0.050$. Quantitative data were expressed as mean \pm standard deviation (SD) if normally distributed or median if not. Progression free survival (PFS) and overall survival (OS) were analyzed by the Kaplan Meier curves and calculated from 
the first day of treatment to tumor recurrence (PFS) or death or last visit (OS).

\section{Results}

A total of 47 patients with muscle-invasive bladder cancer were involved in this study. The patients and tumor characteristics are shown in Table 1.

There were35(74.5\%)males and 12(25.5\%)females, The median age was 65 years (48-72). Most of patients were of ECOG performance status PS1 (44.7\%), followed by PS $2(31.9 \%)$. Tumor stage of T3b $(21 / 44.7 \%)$ was the commonest followed by T4a (16/34\%), also, grade III was the commonest grade $(26 / 55.3 \%)$. All patients underwent maximum TURBT, $16(34.04 \%)$ patients underwent complete TURBT, while 31(65.96\%) underwent incomplete TURBT.

The median follow-up period was 37 months (11-52). Of the47 patients, $25(53.2 \%)$ patients expressed complete response (CR), while $22(46.8 \%)$ patients had incomplete response(PR). Patients with $P R$ were referred to salvage cystectomy if they were operable \&resectable.

Most of the patients have completed their radiotherapy course except 5 patients, who received 25-30 sessions.

The median number of chemotherapy cycles was 3 cycles.

The median duration of radiotherapy interruption was 4days (3-8 days).

\subsection{Toxicity}

Acute toxicity was moderate with the hematological toxicity, was the main toxicity observed during induction chemotherapy, with Grade 2 and 3 toxicities were $42.6 \%$ and $17 \%$, respectively (Table 2 ).

Chemoradiotherapy toxicity affected genitourinary (GU) and gastrointestinal (GI) systems, GU toxicity of Grade 1 and 2 occurs in $26(55.3 \%$ ) and $12(25.5 \%$ ) patients, respectively, while acute gastrointestinal (GI) toxicity(colic\&diarrhea) of Grade 1 and 2 occurs in $27.7 \% \quad 10.6 \%$ of patients, respectively(Table 2).

Late toxicities were mostly of grade $1 \& 2$ with no grade3 or 4 toxicity. Nine $(19.2 \%) \& 5(10.6 \%)$ patients experienced Grade $1 \& 2$ chronic genitourinary toxicity, whereas grade1 and 2GI toxicity were detected in $5(8.5 \%)$ and $2(4.3 \%)$ patients, respectively.

\subsection{Survival}

The 4-year OS, PFS, and LPFS rates were 48\%, 38\%, and $42 \%$, respectively (Figure 1, 2, 3).

On multivariate analysis, ECOG-PS was the independent prognostic factors for OS $(\mathrm{p}=0.03)$, while tumor stage was the independent prognostic factors for PFS \& LPFS ( $p=$ $0.05, \mathrm{p}=0.02$ ).

Table 1. Patients characteristics.

\begin{tabular}{|c|c|}
\hline Characteristics & No $(\%)$ \\
\hline \multicolumn{2}{|l|}{ Age (years) } \\
\hline Median & 65 \\
\hline Range & $(48-72)$ \\
\hline \multicolumn{2}{|l|}{ Sex } \\
\hline Male & $35(74.5 \%)$ \\
\hline Female & $12(25.5 \%)$ \\
\hline \multicolumn{2}{|c|}{ ECOG performance status } \\
\hline 1 & $21(44.7 \%)$ \\
\hline 2 & $15(31.9 \%)$ \\
\hline \multicolumn{2}{|l|}{ Tumor stage } \\
\hline $\mathrm{T} 2$ & $10(21.3 \%)$ \\
\hline $\mathrm{T} 3 \mathrm{~b}$ & $21(44.7 \%)$ \\
\hline $\mathrm{T} 4 \mathrm{a}$ & $16(34 \%)$ \\
\hline \multicolumn{2}{|l|}{ Tumor grade } \\
\hline \multicolumn{2}{|l|}{ TURBT } \\
\hline Complete & $16(34.04 \%)$ \\
\hline incomplete & $31(65.96 \%)$ \\
\hline
\end{tabular}

Table 2. Treatment-related acute toxicity.

\begin{tabular}{|c|c|c|c|c|c|c|c|c|}
\hline \multirow{2}{*}{ Toxicities } & \multicolumn{4}{|c|}{ Neoadjuvant chemotherapy } & \multicolumn{4}{|c|}{ Conc chemoradiotherapy } \\
\hline & I & II & III & IV & I & II & III & IV \\
\hline Hematologic anemia & $16(34.04 \%)$ & $9(19.2 \%)$ & $5(10.6 \%)$ & 0 & $13(27.7 \%)$ & $6(12.8 \%)$ & $3(6.4 \%)$ & 0 \\
\hline leucopenia & $13(27.7 \%)$ & $6(12.8 \%)$ & $3(6.4 \%)$ & 0 & $10(21.3 \%)$ & $5(10.6 \%)$ & 0 & 0 \\
\hline thrombocytopenia & $12(25.5 \%)$ & $5(10.6 \%)$ & 0 & 0 & $8(17.02 \%)$ & $4(8.5 \%)$ & 0 & 0 \\
\hline Non-hematologic GU toxicity & 0 & 0 & 0 & 0 & $26(55.3 \%)$ & $12(25.5 \%)$ & 0 & 0 \\
\hline GI toxicity Nausea, vomiting & $20(42.6 \%)$ & $8(17.02 \%)$ & 0 & 0 & $9(19.2 \%)$ & $4(8.5 \%)$ & 0 & 0 \\
\hline Colic, diarrhea & $7(14.9 \%)$ & $3(6.4 \%)$ & 0 & 0 & $13(27.7 \%)$ & $5(10.6 \%)$ & 0 & 0 \\
\hline
\end{tabular}

GU:genitourinary, GI:gastrointestinal. 


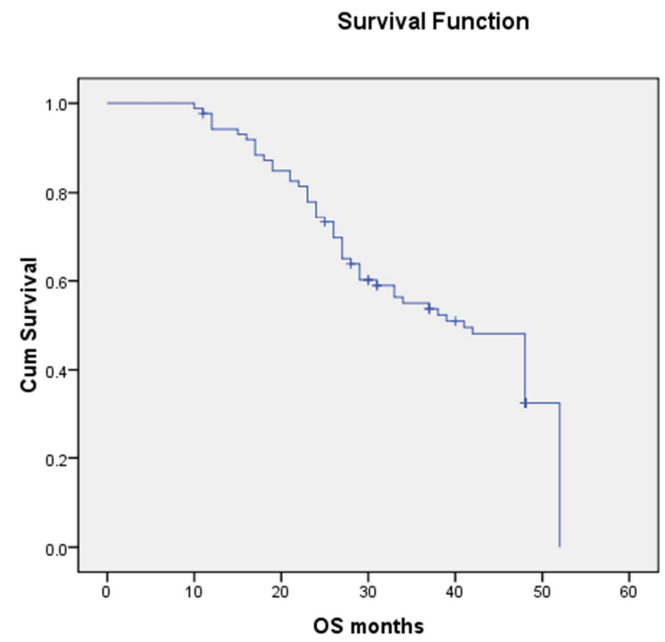

Figure 1. Overall survival curve.

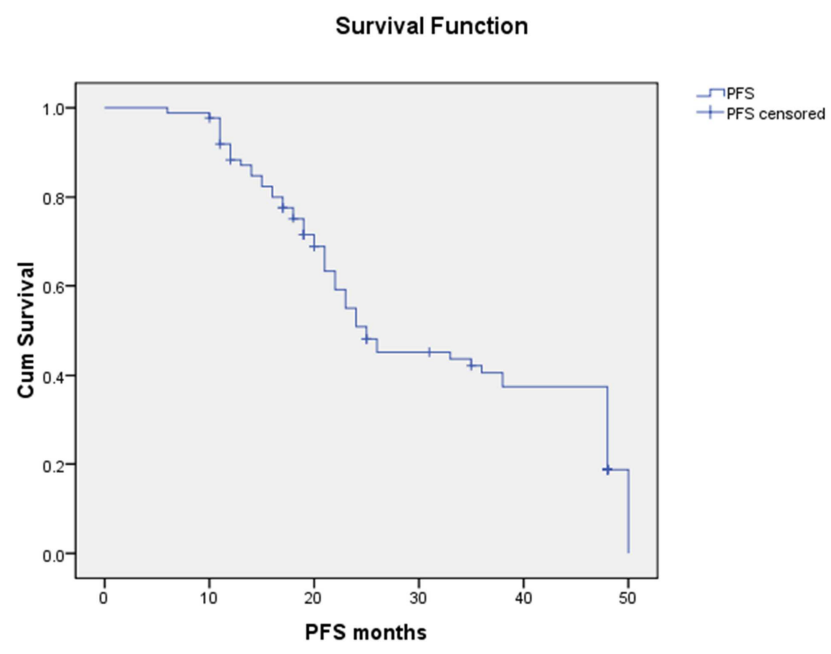

Figure 2. Progression free survival curve.

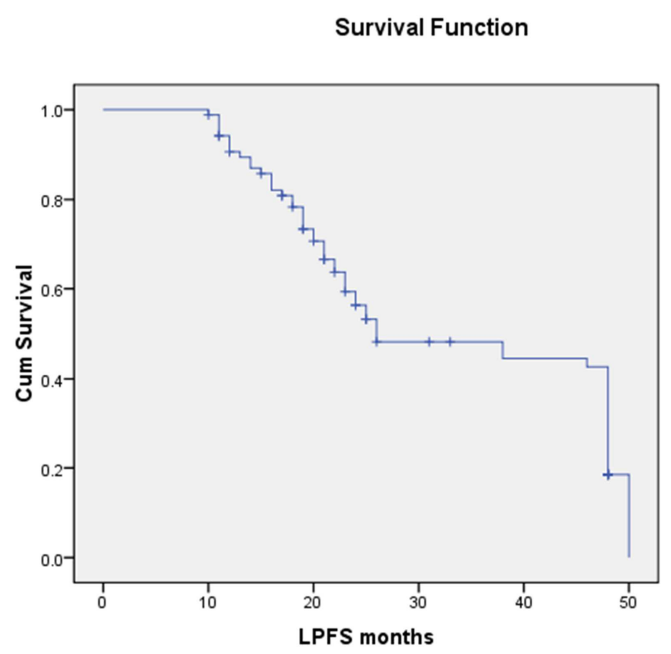

Figure 3. LocalProgression free survival curve.

\section{Discussion}

Platinum based neoadjuvant chemotherapy followed by radical cystectomy is the gold standard therapy for MIBC.
Patients with bladder cancer are older and are often frail, with comorbidities. As a greater percentage of the worldwide population ages, bladder cancer patients will continue to present at advanced age and may no longer be considered ideal candidates for RC, bladder preservation therapy represents a treatment option fornon cystectomy candidates patients [16].

In the current study, Of the 47 patients, 25 (53.2\%) patients expressed complete response (CR), while 22(46.8\%) patients had incomplete response. The 4-year OS, PFS, and LPFS rates were $48 \%, 38 \%$, and $42 \%$, respectively. Acute GU toxicity of Grade $1 \& 2$ occurred in $54 \%$ and $24 \%$ of patients, respectively, while acute GI toxicity (colic \& diarrhea) of Grade 1 \& 2 was detected in $27.7 \%$ and $10.6 \%$ of patients, respectively.

In the BC2001 trial, they targeted by radiotherapy the bladder only. Pelvic lymph node relapse rate was $5 \%$ in the chemoradiotherapy group, which is comparable to a large RC series [17], local relapse in lymph node negative disease was $6 \% \& 13 \%$ for localized and extravesical tumors, respectively. Unplanned involvement of pelvic lymph nodes in the planning target volume may give an explanationof the low incidence of lymph node recurrence. Also, concomitant chemotherapy may have a role in targeting nodal micrometastatic disease [18].

In a randomized study that compared pelvic lymph nodes irradiation (45Gy plus 20 Gy bladder boost) to localized bladder irradiation ( $65 \mathrm{~Gy})$, the incidence of lymph node recurrence in patients who achieved complete response was comparable in both arms (15.8\% versus $17.6 \%)$ [39]. Similarly, OS was $51 \%$ versus $52.9 \%$. Higher incidence of pelvic lymph nodes recurrence in comparison to BC2001 is mostly because half of the patients were of stage T3 but in BC2001 trial, it was only15\% [19].

From this evidence, there is no strong support for pelvic nodal irradiation in clinically node negative disease, so in the current trial, we targeted only the bladder without pelvic lymph nodes involvement depending upon the use of neoadjuvant systemic chemotherapy and concurrent chemoradiotherapy.

In several prospective trials, trimodality therapy demonstrated 5-year OS rates of $48 \%-65 \%$, similar to that registered in RC trials, with approximately $75 \%-80 \%$ of patients maintained their bladders, with good function and better quality of life [20].

A meta-analysis of clinical trials assessing trimodal therapy composed of TURBT followed by chemoradiotherapy in treatment of MIBC, recorded a complete response in $78 \%$ of patients, with $56 \% 5$-year OS [12].

Analysis of Radiation Therapy Oncology Group protocols of bladder preserving therapy using TURBT and concurrent platinum-based chemoradiotherapy revealed a complete response in $69 \%$ of patients, with 5 - \& 10 -year OS of $57 \%$ and $36 \%$, respectively [11].

At the Massachusetts General Hospital between 1986 and 
2013, 475 patients with cT2-T4a MIBC underwent TURBT followed by concurrent chemoradiotherapy. The 5 and 10-yr DSS were $66 \%$ and $59 \%$, respectively, while the 5 - and $10-y$ r OS were $57 \%$ and $39 \%$, respectively. Salvage cystectomy ratewas $29 \%$ at 5 year [15].

In multiple series, the $5 \& 10$-year OS for RCwas $45 \%-60 \%$ and $37 \%-45 \%$, respectively [2-7] while, the 5-year OS for BPT utilizing trimodal therapy was 36-74\% [21-26].

Seventy patients with T2-3N0M0 MIBC received trimodality BPT involving maximum TURBT, small pelvis radiotherapy by proton beam and intra-arterial chemotherapy. The 5-year OS, PFS were $82 \%$ and $77 \%$, respectively. By uni $\&$ multivariate analysis, tumor multiplicity and tumor size larger than or equal to $5 \mathrm{~cm}$ were significant prognostic factors for progression. Regarding toxicity, 26 (18\%) patients expressed G 3 \& 4 acute hematologic toxicity and $2(3 \%)$ patients had G 3 late GUtoxicity [27].

Patients with MIBC T2-3, N0 M0 were randomly assigned to two arms: Arm 1: patients underwent RC alone; and Arm 2, patients underwent maximum TURBT, followed by concomitant chemoradiotherapy. Complete response was found in 62 patients $(83 \cdot 8 \%)$ and residual disease was found in 12 patients $(16 \cdot 2 \%)$. The 3 -year OS for the trimodality group and the RC group were $61 \& 63 \%$, respectively $(p=0 \cdot 425)$, while the DSS were $69 \& 73 \%$, respectively $(\mathrm{p}=0.714)$. The 3 -year OS with intact bladder was $50 \%[28]$.

A total of 112 patients (56 received TMT while 56 underwent RC), TMT had survival outcomes similar to RC. The 5-year disease specific survival was $73.2 \% \& 76.6 \%$ in the RC and TMT arms, respectively $(\mathrm{P}=0.49)$. Cystectomy was done in $10.7 \%$ of patients in the TMT arm [14].

A retrospective analyses published by 2 English cancer centers showed comparable outcomes for RC \& bladder preserving therapy in MIBC patients. Munroe et al showed 10 -year OS of $21.6 \%$ for radiotherapy versus $24.1 \%$ for RCin MIBC [29]. Also, Kotwal et al registered 5-year OS for radical radiotherapy and $\mathrm{RC}$ of $34.6 \%$ and $41.3 \%$, respectively [30].

A case controlled study showed comparable results between RC \& chemoradiotherapy for MIBC [17], 33 patients received chemoradiotherapy because of poor performance for RC were matched with patients of comparable age who underwent RC. The 5- year DFS for RC and chemoradiotherapy were $63.2 \%$ and $54 \%$ respectively, whereas the 5 year OS were $54.8 \%$ \& $56.6 \%$ for RC \& chemoradiotherapy, respectively [13].

A database of 3,024 consecutive patients with clinical T2T4aN0M0 MIBC, from 29 international centers from 2005 to 2013 were enrolled, where265 patients received bladder preserving therapy( BPT) compared to 1,447 patients who underwent $\mathrm{RC}$, BPT patients were older with worse performance status, and more comorbidities $(p<0.01)$. The median OS for BPT and RC were 41 vs 46 months, $\mathrm{p}=0.33$, respectively. Only $4 \%$ of BPT patients underwent salvage cystectomy[31].

Patients who are candidates for bladder preserving therapy
(BPT) are usually older, with worse performance status, and more comorbidities than patients who underwent RC. These conditions greatly direct the treatment decision towards BPT due to lack of ideal candidacy for radical cystectomy. Prior findings similarly showed that non cystectomy candidates have poorer OS when treated with BPT than cystectomy candidates treated with BPT [32].

The survival outcomes in our study and the incidence of complete response were somewhat lower than other trials, which may be explained by the increased incidence of $\mathrm{T} 3 \mathrm{~b}$ $(44.7 \%) \& \mathrm{~T} 4 \mathrm{a}(34 \%)$ with decreased incidence of complete TURBT( $34.04 \%$ ) in our study.

Radical cystectomy (RC) affects quality of life (QOL) significantly despite great advance in surgical techniques because of the presence of a stoma and reduced sexual function [5]. Bladder function and QOL in patients who underwent BPT after 6.3 years of follow up showed $75 \%$ of patients survived with their native bladders and 59\% had satisfactory sexual activity [33].

Another multi-institutional study involved 173 MIBC patients compared QOL in patients treated with BPT and RC, BPTtreated patients was associated with better QOL and bowel function compared to RC, while urinary toxicity was comparable between the 2 treatment modalities [34].

Randomized trials comparing trimodality BPT and RC are needed with comparison of tumor control and QOL.

\section{Conclusion}

For MIBC patients who are non-cystectomy candidates, or who are motivated to keep their native bladders, trimodalitybladder preservation therapy (BPT), including a maximum transurethral resection of the bladder tumor (TURBT), followed by concurrent chemoradiotherapy can be considered as an effective alternative approach.

\section{References}

[1] Siegel RL, Miller KD, Jemal A. Cancer Statistics, 2017. CA Cancer J Clin. 2017;67(1):7.

[2] Ibrahim A, Khaled H, Mikhail N, Baraka H, Kamel H. Cancer Incidence in Egypt: Results of the National Population-Based Cancer Registry Program. Journal of Cancer Epidemiology; Volume 2014, Article ID 437971, 18 pages, http://dx.doi.org/10.1155/2014/437971.

[3] Amin MB, McKenney JK, Paner GP, Hansel DE, Grignon DJ, Montironi R, et al. ICUD-EAU International Consultation on Bladder Cancer 2012: pathology. Eur Urol. 2013;63:16-35.

[4] Mirzaa A, and ChoudhuryaAb. Bladder Preservation for Muscle Invasive Bladder Cancer. Bl Cancer. 2016; 2(2): 151163.

[5] Caffo O, Fellin G, Graffer U, Luciani L. Assessment of quality of life after cystectomy or conservative therapy for patients with infiltrating bladder carcinoma. A survey by a self-administered questionnaire. Cancer 1996;76(9):1089-97. Erratum in: Cancer 1996;76(9):2037. [PubMed]. 
[6] Grossman HB, Natale RB, Tangen CM, Speights VO, Vogelzang NJ, Trump DL, et al. Neoadjuvant chemotherapy plus cystectomy compared with cystectomy alone for locally advanced bladder cancer. N Engl J Med. 2003;349(9):859.

[7] RoseaT, Deala A, Ladoireb S, Cr'ehangeb G, Galskyc M, Rosenbergd J, etal. Patterns of Bladder Preservation Therapy Utilization for Muscle-Invasive. Bladder Cancer.2016; 405413 DOI 10.3233/BLC-160072. IOS Press.

[8] NCCN Guidelines Version 2.2015 Bladder Cancer. http://www.nccn.org/professionals/physician_gls/pdf/bladder. pdf.

[9] Guidelines on Muscle-invasive and Metastatic Bladder Cancer European Association of Urology 2015. http://uroweb.org/wpcontent/uploads/EAU-Guidelines-Muscleinvasive-andMetastatic-Bladder-Cancer-2015-v1.pdf.

[10] Huddart R, Birtle A, Lewis R, Bahl A, Falconer A, MaynardL, et al. Results of the SPARE Feasibility Study; Selective Bladder Preservation Against Radical Excision in Muscle Invasive T2/T3 Transitional Cell Carcinoma of the Bladder. International Journal of Radiation Oncology Biology Physics 2012;84(3):S119-S20.

[11] Mak RH, Hunt D, Shipley WU, Efstathiou JA, Tester WJ, Hagan MP, et al. Long-term outcomes in patients with muscle-invasive bladder cancer after selective bladder preserving combined modality therapy: A pooled analysis of radiation therapy oncology group protocols $8802,8903,9506$, 9706, 9906, and 0233. J ClinOncol 2014;32(34):3801-9.

[12] Arcangeli G, Arcangeli S, Strigari L. A systematic review and meta-analysis of clinical trials of bladder-sparing trimodality treatment of muscle-invasive bladder cancer (MIBC). Crit Rev OncolHematol. 2015;94:105-115.

[13] Gofrit ON, Nof R, Meirovitz A, Pode D, Frank S, Katz R, et al. Radical cystectomy vs. Chemoradiation in T2-4aN0M0 bladder cancer: A case-control study. UrolOncol 2015;33(1):19. [PubMed].

[14] Kulkarni GS, Hermanns T, Wei Y, Bhindi B, Satkunasivam R, Athanasopoulos $\mathrm{P}$, et al. Propensity score analysis of radical cystectomy versus bladder- sparing trimodal therapy in the setting of a multidisciplinary bladder cancer Clinic. J ClinOncol. 2017;35(20):2299-305.

[15] Giacalone NJ, Shipley WU, Clayman RH, Niemierko A, Drumm M, Heney NM, et al. Long-term Outcomes After Bladder-preserving Tri-modality Therapy for Patients with Muscle-invasive Bladder Cancer: An Updated Analysis of the Massachusetts General Hospital Experience. Eur. Urol2017;71, 952-960.

[16] Smelser WW, Austenfeld AM, Holzbeierlein MJ, Lee EK. Where are we with bladder preservation for muscle-invasive bladder cancer in 2017? Indian journal of urology 2017, 33, 2, 111-117.

[17] Stein JP, Lieskovsky G, Cote R, Groshen S, Feng AC, Boyd S, et al. Radical cystectomy in the treatment of invasive bladder cancer: Long-term results in 1,054 patients. J ClinOncol 2001;19(3):666-675. [PubMed].

[18] Pietzak EJ, Sterling ME, Smith ZL, Malkowicz SB, Guzzo TJ. Outcomes of radical cystectomy in potential candidates for bladder preservation therapy. Urology 2015;85(4):869-875. [PubMed].
[19] Tunio MA, Hashmi A, Qayyum A, Mohsin R, Zaeem A. Whole-pelvis or bladder-only chemoradiation for lymph nodenegative invasive bladder cancer: Single-institution experience Int J RadiatOncolBiolPhys 2012;82:457-462. [PubMed].

[20] Chen R, Shipley W, Efstathiou J, and Zietman A. Trimodality Bladder Preservation Therapy for Muscle-Invasive Bladder Cancer. J NatlComprCancNetw2013;11:952-960.

[21] Rödel C, Grabenbauer GG, Kühn R, Papadopoulos T, Dunst J, Meyer M, et al. Combined-modality treatment and selective organ preservation in invasive bladder cancer: long-term results. J ClinOncol 2002;20: 3061-3071. PMID: 12118019.

[22] George L, Bladou F, Bardou VJ, Gravis G, Tallet A, Alzieu C, et al. Clinical outcome in patients with locally advanced bladder carcinoma treated with conservative multimodality therapy. Urology 2004;64: 488-493. doi: 10.1016/j.urology.2004.04.088. PMID: 15351577.

[23] Cobo M, Delgado R, Gil S, Herruzo I, Baena V, Carabante F, et al. Conservative treatment with transurethral resection, neoadjuvant chemotherapy followed by radiochemotherapy in stage T2-3 transitional bladder cancer. Clinical \& Translational Oncology: Official Publication of the Federation of Spanish Oncology Societies and of the National Cancer Institute of Mexico 2006;8(12):903-11. PubMed PMID: 17169764. Epub 2006/12/16.eng. [PubMed].

[24] Weiss C, Engehausen DG, Krause FS, Papadopoulos T, Dunst J, Sauer R, et al. Radiochemotherapy with cisplatin and 5fluorouracil after transurethral surgeryin patients with bladder cancer. Int J Radiat Oncol Biol Phys 2007;68: 1072-1080. doi: 10.1016/j.ijrobp.2007.01.054. PMID: 17467193.

[25] Perdonà S, Autorino R, Damiano R, De Sio M, Morrica $\mathrm{B}$, Meyer M, et al. Bladdersparing, combined-modality approach for muscle-invasive bladder cancer: amulti-institutional, longterm experience. Cancer 2008;112: 75-83. doi: 10.1002/cncr.23137. PMID: 18008364

[26] Efstathiou JA, Spiegel DY, Shipley WU, Heney NM, Kaufman DS, Niemierko A, et al. Long-term outcomes of selective bladder preservation by combined-modality therapy for invasive bladder cancer: the $\mathrm{MGH}$ experience. Eur Urol2011; 61: 705-711. doi: 10.1016/j.eururo.2011.11.010. PMID: 22101114.

[27] Takaoka E, Miyazaki J, Ishikawa H, Kawai K, Kimura T, Ishitsuka $\mathrm{R}$, et al. Long-term single-institute experience with trimodal bladder-preserving therapy with proton beam therapy for muscle-invasive bladder cancer Japanese Journal of Clinical Oncology, Volume 47, Issue 1, 1 January 2017, Pages 67-73.

[28] AlGizawy MS, Essa HH, Abdel-Wanis EM, and Abdel Raheem MA. Trimodality bladder-sparing approach versus radical cystectomy for invasive bladder cancer. Journal of Radiotherapy in Practice Volume 13, Issue 4 December 2014, pp. 428-437.

[29] Munro NP, Sundaram SK, Weston PM, Fairley L, Harrison SC, Forman D, et al. A 10-year retrospective review of a nonrandomized cohort of 458 patients undergoing radical radiotherapy or cystectomy in Yorkshire, UK. Int $\mathrm{J}$ RadiatOncolBiolPhys 2010;77(1):119-124. [PubMed].

[30] Kotwal S, Choudhury A, Johnston C, Paul AB, Whelan P, Kiltie AE. Similar treatment outcomes for radical cystectomy and radical radiotherapy in invasive bladder cancer treated at a United Kingdom specialist treatment center. Int J RadiatOncolBiolPhys 2008;70(2):456-463. [PubMed]. 
[31] Rose LT, Deal AM, Ladoire S, Créhange G, Matthew D. Galsky M, et al the Retrospective International Study of Cancers of the Urothelial Tract (RISC) Investigators. Patterns of Bladder Preservation Therapy Utilization for MuscleInvasive Bladder Cancer. Bladder Cancer. 2016; 2(4): 405413.

[32] Hussain MH, Glass TR, Forman J, Sakr W, Smith DC, AlSarraf M, et al. Combination cisplatin, 5-fluorouracil and radiation therapy for locally advanced unresectable or medically unfit bladder cancer cases: A Southwest Oncology Group Study. J Urol2001;165(1):56-60; discussion -1. [PubMed].
[33] Zietman AL, Sacco D, Skowronski U, Gomery P, Kaufman DS, Clark JA, et al. Organ conservation in invasive bladder cancer by transurethral resection, chemotherapy and radiation: Results of a urodynamic and quality of life study on long-term survivors. J Urol 2003;170(5):1772-1776.[PubMed].

[34] Mak KS, Smith AB, Eidelman A, Clayman R, Niemierko A, Cheng JS, et al. Quality of life in long-term survivors of muscle-invasive bladder cancer. J ClinOncol 2015;33(supp1 7; abstr 319). 